\title{
Correction to: Evaluating the therapeutic potential of ADAR1 inhibition for triple-negative breast cancer
}

Che-Pei Kung 1 - Kyle A. Cottrell • Sua Ryu • Emily R. Bramel • Raleigh D. Kladney • Emily A. Bao • Eric C. Freeman • Thwisha Sabloak • Leonard Maggi Jr. (1D - Jason D. Weber (D)

Published online: 9 February 2021

(c) The Author(s), under exclusive licence to Springer Nature Limited 2021

\section{Correction to: Oncogene}

https://doi.org/10.1038/s41388-020-01515-5

The original version of this article unfortunately contained a mistake. The following correction has therefore been made in the original: The word "orthopedic" was changed to "orthotopic" in subheading "Mammary gland orthotopic implantation" and in the first sentence of section "Mammary gland orthotopic implantation": "The abilities of human breast cancer cell lines to form tumors in vivo were evaluated by performing mammary gland orthotopic implantation as described previously, further details can be found in the Supplementary Information [48]."

The original article has been corrected. 